\title{
УРАВНЕНИЯ КИРКВУДА - ЗАЛЬЦБУРГА ДЛЯ РЕШЕТЧАТЫХ КЛАССИЧЕСКИХ МОДЕЛЕЙ СТАТИСТИЧЕСКОЙ МЕХАНИКИ
}

\section{Ю. П. Вирченко, Е. Ю. Московченко}

Белгородский государственный национальный исследователький университет, г. Белгород, 308015, Россия

E-mail:virch@bsu.edu.ru

\begin{abstract}
Аннотация. Изучается класс решетчатых моделей статистической механики классических систем с суммируемым парным потенциалом взаимодействия, которые с физической точки зрения описывают т.н. разбавленные системы многих частиц. Получена система уравнений для частных распределений вероятностей, аналогичная системе уравнений Кирквуда - Зальцбурга, которая применяется для исследования непрерывных систем.
\end{abstract}

Ключевые слова: статистическая механика, распределения Гиббса, решетчатые системы, уравнения Кирквуда-Зальцбурга, статистическая сумма, термодинамический предел, гамильтониан, периодические условия. Для цитирования: Вирченко Ю. П., Московченко Е. Ю. 2020. Уравнения Кирквуда - Зальбурга для решетчатых классических моделей статистической механики. Прикладная математика \& Физика, $52(2)$ : 62-70. DOI 10.18413/2687-0959-2020-52-2-62-70.

\section{KIRKWOOD - SALZBURG EQUATIONS FOR LATTICE CLASSICAL MODELS OF STATISTICAL MECHANICS}

\author{
Yu. P. Virchenko, E. Yu. Moskovchenko \\ Belgorod National Research University, \\ Belgorod, 308015, Russia \\ E-mail: virch@bsu.edu.ru
}

Received February 1, 2020

\begin{abstract}
Lattice models of statistical mechanics of classical systems with a summable pair interaction potential, which from the physical point of view describe the so-called diluted systems of many particles are studied. The equations system of partial probabilities is obtained that is similar to the Kirkwood - Salzburg system which is used when continuous models are studied.
\end{abstract}

Key words: statistical mechanics, Gibbs distributions, lattice systems, Kirkwood - Salzburg equations, partition function, thermodynamic limit, hamiltonian, periodic conditions.

For citation: Virchenko Yu. P., Moskovchenko E. Yu. 2020. Kirkwood - Salzburg's equations for lattice classical models of statistical mechanics. Applied Mathematics \& Physics, 52(2): 62-70 (in Russian).

DOI 10.18413/2687-0959-2020-52-2-62-70.

Введение. Математическим объектом изучения в равновесной статистической механике являются гиббсовские вероятностные меры. Частным случаем таких мер, который изучается в настоящей работе, являются гиббсовские меры, связанные с т. н. решетчатыми моделями, которые являются математическими моделями систем многих частиц, рассматриваемых в физике твердого тела. Для решетчатых моделей меры определяются посредством задания семейства согласованных между собой частных распределений вероятностей $\mathrm{P}_{\Lambda}[\cdot]$ на пространствах элементарных событий $\Omega(\Lambda)$, каждое из которых сопоставляется множеству $\Lambda \subset \mathbb{Z}^{d},|\Lambda|<\infty, d=1,2,3$ (конечной части кристаллической решетки), принадлежащему специальному классу конечных подмножеств из $\mathbb{Z}^{d}$. Тогда гиббсовская мера $\mathrm{P}[\Sigma]$ случайного события $\Sigma$, связанного с фиксированным конечным множеством $\Lambda \subset \mathbb{Z}^{d}$ определяется как совокупность предельных значений последовательности $\left\langle\mathrm{P}_{\Lambda}[\Sigma] ; \Lambda \subset \mathbb{Z}^{d}\right\rangle$, $\Sigma \subset \Omega(\Lambda)$, которая соответствует расширяющейся последовательности множеств $\Lambda \rightarrow \mathbb{Z}^{d}$. Такие предельные значения вероятностей называются термодинамически предельными вероятностями. По поводу используемой терминологии (см., например, [Минлос, 2002], [Gallavotti, 1999]).

Одной из задач статистической механики является задача вычисления предельных значений $P[\Sigma]$ и один из подходов к решению этой задачи связывается с нахождением подходящей системы 
уравнений, связывающих предельные значения $\mathrm{P}[\Sigma]$ различных случайных событий $\Sigma$. В простейшем случае решетчатых моделей, которые соответствуют гиббсовским точечным случайным полям и называются «решеточным газом», такой системой уравнений являются интегральные уравнения, которые применялись для исследования таких моделей в работах [Gallavotti, Miracle-Sole, 1967], [Добрушин, 1968], а также ее видоизменение в работе [Пастур, 1974]. В последнем случае система уравнений аналогична системе уравнений Кирквуда - Зальцбурга [Kirkwood, Salsburg, 1953], используемой при изучении непрерывных моделей статистической механики. Настоящая работа посвящена выводу системы интегральных уравнений, которая представляет собой обобщение систем уравнений, полученных в цитируемых работах, на случай решетчатых систем, которые представляют собой векторные расслоения гиббсовских точечных случайных полей. Кроме того, мы распространим положения спектральной теории Л. А. Пастура для полученной нами системы уравнений.

2. Векторные решетчатые модели статистической механики. Определим для каждого множества $\Lambda$ вероятностные пространства $\left\langle\mathfrak{S}(\Lambda), \mathrm{P}_{\Lambda}\right\rangle$ решетчатых систем статистической механики, изучаемых в настоящей работе, где $\mathfrak{S}(\Lambda)$ - пространство состояний системы (пространство элементарных случайных событий) и $\mathrm{P}_{\Lambda}-$ нормированная мера, заданная в соответствии со структурой измеримости на $\Omega(\Lambda)$.

Прежде всего, опишем класс подмножеств $\Lambda(L) \equiv \Lambda \subset \mathbb{Z}^{d}$. Определим для любого $L \in \mathbb{N}_{+}$ множество $\Lambda=\{0,1, \ldots, L\}^{d}-a_{L}\langle 1, \ldots, 1\rangle$ с $a_{L}=L / 2$, если $L$ - четное, и $(L-1) / 2$, если $L$ - нечетное. При этом для любого $L$ имеет место включение $\Lambda(L+1) \supset \Lambda(L)$ и $\bigcup_{L=0}^{\infty} \Lambda(L)=\mathbb{Z}^{d}$. Число $L$ будем называть размером множества $\Lambda$. При этом число точек в множестве $\Lambda$ с размером $L$ равно $|\Lambda| \equiv(L+1)^{d}$.

Пространства состояний решетчатых систем статистической механики для каждого из указанных выше множеств $\Lambda$ представляются в виде прямого произведения

$$
\mathfrak{S}(\Lambda)=\bigotimes_{\mathbf{x} \in \Lambda} \mathfrak{S}(\{\mathbf{x}\})
$$

Подразумевается, что на пространстве состояний $\mathfrak{S}_{\Lambda}$ имеется структура измеримости и на ней определен интеграл по $\sigma$-аддитивной мере. При этом измеримые множества определяются как прямые произведения измеримых множеств в каждом из пространств $\mathfrak{S}(\{\mathbf{x}\})$ состояний с $\mathbf{x} \in \Lambda$, а мера на $\mathfrak{S}(\Lambda)$ определяется как произведение мер $\prod_{\mathbf{x} \in \Lambda} d m_{\mathbf{x}}$, где входящие в это произведение меры $\mathrm{dm}_{\mathbf{x}}, \mathbf{x} \in \Lambda$ эквивалентны. Тогда гиббсовские распределения вероятностей $\mathrm{P}_{\Lambda}[\Sigma]$ случайных событий $\Sigma \subset \mathfrak{S}(\Lambda)$ для систем статистической механики определяются, для каждого $\Lambda$, на основе задания функционала $\mathrm{H}_{\Lambda}[\cdot]$ на пространстве состояний $\mathfrak{S}(\Lambda)$ посредством формулы

$$
\begin{aligned}
\mathrm{P}_{\Lambda}[\Sigma]=Q_{\Lambda}^{-1} \int_{\Sigma} \exp \left(-\mathrm{H}_{\Lambda} / T\right) & \prod_{\mathbf{x} \in \Lambda} \mathrm{dm}_{\mathbf{x}}, \\
Q_{\Lambda} & =\int_{\mathfrak{S}_{\Lambda}} \exp \left(-\mathrm{H}_{\Lambda} / T\right) \prod_{\mathbf{x} \in \Lambda} \mathrm{dm}_{\mathbf{x}} .
\end{aligned}
$$

Здесь параметр $T>0$, называемый температурой. Функционал $\mathrm{H}_{\Lambda}[\cdot]$ называется гамильтонианом системы.

Для рассматриваемых нами в этой работе векторных решетчатых моделей пространство состояний $\mathfrak{S}(\{\mathbf{x}\})$ в каждой точке $\mathbf{x} \in \Lambda$ определяется формулой

$$
\mathfrak{S}(\{x\})=\left\{\langle\rho(\mathbf{x}), \mathbf{s}(\mathbf{x})\rangle: \rho(\mathbf{x}) \in\{0,1\}, \mathbf{s}(\mathbf{x}) \in \mathbb{R}^{n}, \mathbf{s}^{2}(\mathbf{x}) \leq s^{2}\right\},
$$

$s \in(0, \infty), n \in \mathbb{N}$ так, что все пространство $\mathfrak{S}(\Lambda)$ составляют множество пар $\langle\rho(\mathbf{x}), \mathbf{s}(\mathbf{x})\rangle$ функций на $\Lambda$, из которых $\rho(\mathbf{x})$ - дихотомическая функция со значениями $\{0,1\}$ и $\mathbf{s}(\mathbf{x})$ - векторное поле на $\Lambda$ со значениями в $\mathbb{R}^{n}, n=1,2,3$. Следовательно, элементами пространства $\mathfrak{S}(\Lambda)$ являются пары $\langle\rho(\mathbf{x}), \mathbf{s}(\mathbf{x})\rangle$ и гамильтониан $\mathrm{H}_{\Lambda}[\cdot]$ сопоставляет каждой такой паре число из $\mathbb{R}$. Поэтому его значения мы будем, далее, обозначать посредством $\mathrm{H}_{\Lambda}[\rho, \mathbf{s}]$.

В свою очередь, для векторных моделей измеримые множества в каждом из пространств $\mathfrak{S}(\{\mathbf{x}\})$, $\mathbf{x} \in \Lambda$ определяются измеримыми по Лебегу множествами в $\mathbb{R}^{n}$ как при значении $\rho(\mathbf{x})=0$, так и при значении $\rho(\mathbf{x})=1$, а мера $\mathrm{dm}[\mathbf{s}(\mathbf{x})]$ на каждом из этих пространств определяется сферически симметричной плотностью $f(s)$, сосредоточенной на $[0, s]$ так, что для фиксированной точки $\mathbf{x} \in \Lambda$ дифференциал меры множества точек пространства $\mathfrak{S}(\{\mathbf{x}\})$ для каждого значения $\rho=\rho(\mathbf{x}) \in\{0,1\}$, составляющий дифференциальную часть $s^{n-1} d s d \Omega$ сферического слоя около точки $\mathbf{s} \in \mathbb{R}^{n} \mathrm{c}|\mathbf{s}|=s$, определяемую дифференциалом телесного угла $d \Omega$, равен $w(s) s^{n-1} d s d \Omega \equiv w(s) d \mathbf{s}$. Таким образом, 
в соответствии с формулами (2), (3), гиббсовское распределение вероятностей для измеримых множеств $\Sigma$ в пространстве $\mathfrak{S}(\Lambda)$ векторных моделей определяется следующим образом:

$$
\begin{aligned}
\mathrm{P}_{\Lambda}[\Sigma]=Q_{\Lambda}^{-1} \sum_{\substack{\rho(\mathbf{x}) \in\{0,1\}^{\Lambda} \\
\langle\rho, \mathbf{s}\rangle \in \Sigma}} \int_{\Sigma_{\mathbf{s}}} \exp \left(-\mathrm{H}_{\Lambda}[\rho, \mathbf{s}] / T\right) \prod_{\mathbf{x} \in \Lambda} w(s(\mathbf{x})) \mathrm{d} \mathbf{s}(\mathbf{x}), \\
Q_{\Lambda}=\sum_{\rho(\mathbf{x}) \in\{0,1\}^{\Lambda}} \int_{\left(\mathbb{R}^{n}\right)^{|\Lambda|}} \exp \left(-\mathrm{H}_{\Lambda}[\rho, \mathbf{s}] / T\right) \prod_{\mathbf{x} \in \Lambda} w(s(\mathbf{x})) \mathrm{d} \mathbf{s}(\mathbf{x}),
\end{aligned}
$$

где $|\mathbf{s}(\mathbf{x})|=s(\mathbf{x})$ и введено обозначение $\Sigma_{\mathbf{s}}=\{\langle\mathbf{s}(\mathbf{x}) ; \mathbf{x} \in \mathbf{\Lambda}\rangle:\langle\langle\rho(\mathbf{x}), \mathbf{s}(\mathbf{x})\rangle ; \mathbf{x} \in \mathbf{\Lambda}\rangle \in \mathbf{\Sigma}\}$.

Далее, в этой работе мы будем исследовать векторные модели, гамильтонианы которых содержат только парное взаимодействие между точками $\mathbf{x} \in \Lambda$. Такого рода функционалы определяются формулой

$$
\mathbf{H}_{\Lambda}[\rho(\mathbf{x}), \mathbf{s}]=-\sum_{\mathbf{x} \in \Lambda} \rho(\mathbf{x})(\mathbf{s}(\mathbf{x}), \mathbf{h})+\frac{1}{2} \sum_{\mathbf{x}, \mathbf{y} \in \Lambda^{2}} U(\mathbf{x}-\mathbf{y}) \rho(\mathbf{x}) I(\mathbf{s}(\mathbf{x}), \mathbf{s}(\mathbf{y})) \rho(\mathbf{y}) .
$$

Здесь в первом слагаемом $(\mathbf{s}(\mathbf{x}), \mathbf{h})$ обозначает скалярное произведение вектора $\mathbf{s}(\mathbf{x})$ и постоянного вектора $\mathbf{h}$. Функция $U(\cdot): \mathbb{Z}^{d} \mapsto \mathbb{R}$ обладает свойством $U(-\mathbf{x})=U(\mathbf{x}), U(0)=0$ и является суммируемой $\sum_{\mathbf{x} \in \mathbb{Z}^{d}}|U(\mathbf{x})|<\infty$. Кроме того, функция $I\left(\mathbf{s}_{1}, \mathbf{s}_{2}\right)$ является симметричной относительно перестановок аргументов, ограниченной $\left|I\left(\mathbf{s}_{1}, \mathbf{s}_{2}\right)\right| \leq I, \mathbf{s}_{j} \in \mathbb{R}^{n}, j=1,2$ некоторой постоянной $I>0$. Функция $I\left(\mathbf{s}_{1}, \mathbf{s}_{2}\right)$ предполагается зависящей только от инвариантов пары векторов $\left\langle\mathbf{s}_{1}, \mathbf{s}_{2}\right\rangle$, то есть от $\mathbf{s}_{1}^{2}, \mathbf{s}_{2}^{2}$ и скалярного произведения $\left(\mathbf{s}_{1}, \mathbf{s}_{2}\right)$.

Для каждого $n=1 \div|\Lambda|$ и непустого множества $X \subset \Lambda,|X|=m$ и связанного с ним набора $\left\langle\Sigma_{\mathbf{s}(\mathbf{x})} ; \mathbf{x} \in X\right\rangle$ рассмотрим вероятности

$$
\begin{aligned}
\operatorname{Pr}\left\{\tilde{\rho}(\mathbf{x})=1 \vee \tilde{\mathbf{s}}(\mathbf{x}) \in \Sigma_{\mathbf{s}(\mathbf{x})} ; \mathbf{x} \in X\right\}=Q_{\Lambda}^{-1} \sum_{\rho \in\{0,1\}^{\Lambda}}\left(\prod_{\mathbf{x} \in X} \rho(\mathbf{x})\right) \times \\
\times \int_{\substack{\mathbf{s}(\mathbf{x}) \in \Sigma_{\mathbf{s}(\mathbf{x})} ; \\
\mathbf{x} \in X}} \int_{\substack{\mathbf{s}(\mathbf{x}) \in \mathbb{R}^{n} ; \\
\mathbf{x} \in \Lambda \backslash X}} \exp \left(-\mathbf{H}_{\Lambda}[\rho, \mathbf{s}] / T\right) \prod_{\mathbf{y} \in \Lambda} w(s(\mathbf{y})) \mathrm{d} \mathbf{s}(\mathbf{y}) .
\end{aligned}
$$

Здесь знаком «тильда» помечены случайные величины.

Введем плотности $f_{m}(X ; \mathbf{s}(\mathbf{x}), \mathbf{x} \in X)$ распределения этих вероятностей, которые выражаются производными по мере $\prod_{\mathbf{x} \in X} \mathrm{~d} \mathbf{s}(\mathbf{x})$ множества $\Sigma_{\mathbf{s}}$,

$$
\begin{aligned}
f_{m}^{(\Lambda)}(X ; \mathbf{s}(\mathbf{x}), \mathbf{x} \in X)=Q_{\Lambda}^{-1} & \sum_{\rho \in\{0,1\}^{\Lambda}}\left(\prod_{\mathbf{x} \in X} \rho(\mathbf{x}) w(s(\mathbf{x}))\right) \times \\
& \times \int_{\substack{\mathbf{s}(\mathbf{y}) \in \mathbb{R}^{n}: \\
\mathbf{y} \in \Lambda \backslash X}} \exp \left(-\mathbf{H}_{\Lambda}[\rho, \mathbf{s}] / T\right) \prod_{\mathbf{y} \in \Lambda \backslash X} w(s(\mathbf{y})) \mathrm{d} \mathbf{s}(\mathbf{y}) .
\end{aligned}
$$

Тогда каждая из вероятностей $p(X)=\operatorname{Pr}\{\tilde{\rho}(\mathbf{x})=1 ; \mathbf{x} \in X\}, \varnothing \neq X \subset \Lambda$ определяется формулой

$$
\begin{gathered}
p(X)=\sum_{\rho \in\{0,1\}^{\Lambda}} \int_{\substack{\mathbf{s}(\mathbf{y}) \in \mathbb{R}^{n}: \\
\mathbf{y} \in X}} f_{m}^{(\Lambda)}(X ; \mathbf{s}(\mathbf{x}), \mathbf{x} \in X) \prod_{\mathbf{y} \in X} w(s(\mathbf{y})) \mathrm{d} \mathbf{s}(\mathbf{y})= \\
=Q_{\Lambda}^{-1} \sum_{\rho \in\{0,1\}^{\Lambda}}\left(\prod_{\mathbf{x} \in X} \rho(\mathbf{x}) w(s(\mathbf{x}))\right) \int_{\substack{\mathbf{s}(\mathbf{y}) \in \mathbb{R}^{n}: \\
\mathbf{y} \in \Lambda \backslash X}} \exp \left(-\mathrm{H}_{\Lambda}[\rho, \mathbf{s}] / T\right) \prod_{\mathbf{y} \in \Lambda \backslash X} w(s(\mathbf{y})) \mathrm{d} \mathbf{s}(\mathbf{y}) .
\end{gathered}
$$

Формулу (7) можно записать в иной форме, более удобной для решения той задачи, которой посвящена настоящая работа. Сопоставим каждой функции $\rho(\mathbf{x})$, множество $Z=\{\mathbf{z} \in \Lambda: \rho(\mathbf{z})=1\}$, в терминах которого запишем формулу (6) для гамильтониана системы

$$
\mathrm{H}_{\Lambda}[\rho(\mathbf{x}), \mathbf{s}] \equiv H_{\Lambda}(Z ; \mathbf{s})=-\sum_{\mathbf{z} \in Z}(\mathbf{s}(\mathbf{z}), \mathbf{h})+\frac{1}{2} \sum_{\langle\mathbf{x}, \mathbf{y}\rangle \in Z} U(\mathbf{x}-\mathbf{y}) I(\mathbf{s}(\mathbf{x}), \mathbf{s}(\mathbf{y})) .
$$


Плотность $f_{m}^{(\Lambda)}(X ; \mathbf{s}(\mathbf{x}), \mathbf{x} \in X)$ в терминах такой функции $H(Z), Z \subset \Lambda$ записывается в виде

$$
\begin{aligned}
& f_{m}^{(\Lambda)}(X ; \mathbf{s}(\mathbf{x}), \mathbf{x} \in X)=Q_{\Lambda}^{-1}\left(\prod_{\mathbf{x} \in X} w(s(\mathbf{x}))\right) \times \\
& \times \sum_{Y \subset \Lambda \backslash X} \int_{\substack{\mathbf{s}(\mathbf{y}) \in \mathbf{\Sigma}_{\mathbf{X}} ; \\
\mathbf{y} \in Y}} \exp \left(-\mathrm{H}_{\Lambda}(X \cup Y ; \mathbf{s}) / T\right) \prod_{\mathbf{y} \in Y} w(s(\mathbf{y})) \mathrm{d} \mathbf{s}(\mathbf{y}), \\
& Q_{\Lambda}=\sum_{X \subset \Lambda} \int_{\mathbf{s}(\mathbf{x}) ; \mathbf{x} \in \mathbf{X}} \exp \left(-\mathrm{H}_{\Lambda}(X ; \mathbf{s}) / T\right) \prod_{\mathbf{x} \in X} w(s(\mathbf{x})) \mathrm{d} \mathbf{s}(\mathbf{x}) .
\end{aligned}
$$

3. Периодические условия. В статистической механике часто применяется аппроксимация, основанная на замене гамильтониана $\mathrm{H}_{\Lambda}$ гамильтонианом $\hat{\mathrm{H}}_{\Lambda}$, получаемом в результате отождествления противоположных граней параллелепипеда $\Lambda$, на котором определено пространство $\mathfrak{S}(\Lambda)$ состояний модели. Получаемая при этом модель называется моделью с периодическими граничными условиями, соответствующей исходной решеточной модели (см. [Минлос, 2002]). Использование модели с периодическими граничными условиями упрощает всевозможные конструкции в рамках статистической механики, связанные с вычислениями статистических и термодинамических характеристик модели и доказательства утверждений о ее качественных свойствах.

Обычно, понятие системы с периодическими граничными условиями вводится в том случае, когда взаимодействие обладает конечным радиусом [Минлос, 2002]. Однако при оценке энергии конкретного состояния, в частности, при решении задачи об определении основного состояния конечной системы статистической механики, совсем не очевидно, что аппроксимация исходного гамильтониана $\mathrm{H}_{\Lambda}[\cdot]$ системы, который физически не обладает конечным радиусом действия, каким-либо гамильтонианом конечного радиуса действия, приводит к близости вычисляемых величин.

В этом разделе мы вводим для каждой модели, определяемой гамильтонианом (6), понятие аппроксимирующей ее модели с периодическими граничными условиями в том случае, когда потенциал $U$ не обладает конечным радиусом действия, и находим оценку близости энергий исходной и аппроксимирующей моделей для каждого состояния из $\mathfrak{S}(\Lambda)$.

Зафиксируем множество $\Lambda$ и на его основе определим для каждой точки $\mathbf{x} \in \mathbb{Z}^{d}$ действие оператора $\mathrm{P}_{\Lambda}$ проектирования. Точка $\mathbf{x} \in \mathbb{Z}^{d}$ однозначно представима в виде $\mathbf{x}=(L+1) \sum_{j=1}^{d} n_{j} \mathbf{e}_{j}+\mathbf{y}$, $\mathbf{y} \in \Lambda, \mathbf{e}_{j}$ - орты в $\mathbb{R}^{d},\left(\mathbf{e}_{j}\right)_{i}=\delta_{i j}, n_{j} \in \mathbb{Z}, i, j=1 \div d$. Положим, по определению, $\mathrm{P}_{\Lambda} \mathbf{x}=\mathbf{y}$.

Определение. Гамильтониан

$$
\hat{\mathrm{H}}_{\Lambda}[\rho, \mathbf{s}]=-\sum_{\mathbf{x} \in \Lambda} \rho(\mathbf{x})(\mathbf{s}(\mathbf{x}), \mathbf{h})+\frac{1}{2} \sum_{\substack{\mathbf{x} \in \Lambda, \mathbf{y} \in \mathbb{Z}^{d}, \mathbf{y} \neq \mathbf{x}}} U(\mathbf{x}-\mathbf{y}) \rho(\mathbf{x}) I\left(\mathbf{s}(\mathbf{x}), \mathbf{s}\left(\mathrm{P}_{\Lambda} \mathbf{y}\right)\right) \rho\left(\mathrm{P}_{\Lambda} \mathbf{y}\right)
$$

назовем гамильтонианом с периодическими граничными условиями, аппроксимирующим гамильтониан $\mathrm{H}_{\Lambda}[\rho, \mathrm{s}]$.

Сопоставив каждой функции $\rho(\mathbf{x})$ множество $Z=\{\mathbf{x} \in \Lambda: \rho(\mathbf{x})=1\}$, формулу (11), определяющую гамильтониан с периодическими условиями, запишем в виде

$$
\hat{\mathrm{H}}_{\Lambda}(Z ; \mathbf{s})=-\sum_{\mathbf{x} \in X}(\mathbf{s}(\mathbf{x}), \mathbf{h})+\frac{1}{2} \sum_{\substack{\mathbf{x} \in Z, \mathbf{y} \in \mathbb{Z}^{d}: \mathbf{x} \neq \mathrm{P}_{\Lambda} \mathbf{y} \in Z}} U(\mathbf{x}-\mathbf{y}) I\left(\mathbf{s}(\mathbf{x}), \mathbf{s}\left(\mathrm{P}_{\Lambda} \mathbf{y}\right)\right) .
$$

Нормой $\|\cdot\|_{0}$ гамильтониана $\hat{\mathrm{H}}_{\Lambda}$ называется число

$$
\left\|\mathrm{H}_{\Lambda}[\rho, \mathbf{s}]\right\|_{0}=\max \left\{|\Lambda|^{-1}\left|\mathrm{H}_{\Lambda}[\rho, \mathbf{s}]\right|:\langle\rho(\mathbf{x}), \mathbf{s}(\mathbf{x}) ; \mathbf{x} \in \Lambda\rangle \in \mathfrak{S}(\Lambda), \Lambda \subset \mathbb{Z}^{d}\right\} .
$$

В случае гамильтониана с конечным радиусом действия, очевидно, что разность между энергиями $\mathrm{H}_{\Lambda}[\mathbf{s}]$ и $\hat{\mathrm{H}}_{\Lambda}[\mathbf{s}]$ должна быть пропорциональна площади поверхности кристалла, то есть $L^{d-1}$ при $L \rightarrow \infty$. Если же взаимодействие является дальнодействующим, то такая оценка может быть слабее.

Для получения оценок близости по норме $\|\cdot\|_{0}$ гамильтонианов $\mathrm{H}_{\Lambda}[\rho, \mathbf{s}]$ и $\hat{\mathrm{H}}_{\Lambda}[\rho, \mathbf{s}]$ установим предварительно следующую простую геометрическую оценку

Лемма. Для любой точки $\mathbf{z} \in \mathbb{Z}^{d}$ имеет место следующее неравенство

$$
\left|\Lambda \cap\left(\mathbb{Z}^{d} \backslash \Lambda+\mathbf{z}\right)\right| \leq d L^{d-1} \max \left\{\left|z_{j}\right| ; j=1, \ldots, d\right\} \equiv d L^{d-1}\|\mathbf{z}\|
$$

и при $|\mathbf{z}|>L \sqrt{d}+1$ выполняется $\left|\Lambda \cap\left(\mathbb{Z}^{d} \backslash \Lambda+\mathbf{z}\right)\right|=0$. 
Любая точка $\mathrm{x} \in \Lambda$ после сдвига вдоль одной из осей на величину $L$ приведет к тому, что она либо выйдет за пределы $\Lambda$, либо перейдет в граничную точку $\Lambda$. Тогда сдвиг на любой вектор $\mathbf{z}=L \sum_{j=1}^{d} \theta_{j} \mathbf{e}_{j}, \theta_{j} \in\{0,1\}$ приведет к тому, что она выйдет за пределы $\Lambda$, либо попадет в угловую точку $\Lambda$. Длина вектора $\mathbf{z}$ в этом случае не превосходит $L \sqrt{d}$. Тогда точка $\mathbf{x}$, наверняка, выйдет за пределы $\Lambda$, если $|\mathbf{z}|>L \sqrt{d}$. Ввиду того, что точка $\mathbf{x} \in \Lambda$ выбрана произвольно, то $\Lambda \cap\left(\mathbb{Z}^{d} \backslash \Lambda+\mathbf{z}\right)=\varnothing$, если $|\mathbf{z}| \geq L \sqrt{d}+1$. Отсюда следует последнее равенство в формулировке леммы.

Доказательство неравенства (14) проведем индукцией по $d$. При $d=1$ и $|\mathbf{z}| \leq L$ имеем точное равенство, так как $\Lambda=\{0,1, \ldots, L\}$ и $\Lambda+\mathbf{z}=\{z, z+1, \ldots, z+L\}$. Тогда $|\Lambda \cap(\mathbb{Z} \backslash \Lambda+\mathbf{z})|=L-(L-|z|)=|z|$.

Пусть неравенство (14) имеет место для значения $d$. Тогда, так как, в общем случае,

$$
\left|\Lambda \cap\left(\mathbb{Z}^{d} \backslash \Lambda+\mathbf{z}\right)\right|=(L+1)^{d}-\prod_{j=1}^{d}\left(L+1-\left|z_{j}\right|\right),
$$

то для значения $(d+1)$ и любой точки $\mathbf{z}=\left\langle z_{1}, \ldots, z_{d}, z_{d+1}\right\rangle$, имеем, согласно предположению индукции,

$$
\begin{gathered}
\left|\Lambda \cap\left(\mathbb{Z}^{d+1} \backslash \Lambda+\mathbf{z}\right)\right|=(L+1)^{d+1}-\prod_{j=1}^{d+1}\left(L+1-\left|z_{j}\right|\right)= \\
=(L+1)\left((L+1)^{d}-\prod_{j=1}^{d}\left(L+1-\left|z_{j}\right|\right)\right)+\left((L+1) \prod_{j=1}^{d}\left(L+1-\left|z_{j}\right|\right)-\prod_{j=1}^{d+1}\left(L+1-\left|z_{j}\right|\right)\right) \leq \\
\leq d L^{d} \max \left\{\left|z_{j}\right| ; j=1, \ldots, d\right\}+\left(\prod_{j=1}^{d}\left(L+1-\left|z_{j}\right|\right)\right)\left(L+1-\left(L+1-\left|z_{d+1}\right|\right)\right) \leq \\
\leq d(L+1)^{d} \max \left\{\left|z_{j}\right| ; j=1, \ldots, d\right\}+(L+1)^{d}\left|z_{d+1}\right| \leq(d+1)(L+1)^{d} \max \left\{\left|z_{j}\right| ; j=1, \ldots, d+1\right\} .
\end{gathered}
$$

Из полученной геометрической оценки следует оценка близости энергий произвольного состояния $\langle\rho(\mathbf{x}), \mathbf{s}(\mathbf{x})\rangle, \mathbf{x} \in \Lambda$, вычисленных на основе гамильтонианов $\mathrm{H}_{\Lambda}$ и $\hat{\mathbf{H}}_{\Lambda}$.

Теорема. Имеет место следующее неравенство:

$$
\left\|\mathrm{H}_{\Lambda}[\rho, \mathbf{s}]-\tilde{\mathbf{H}}_{\Lambda}[\rho, \mathbf{s}]\right\|_{0} \leq \frac{d I}{2(L+1)} \sum_{\mathbf{x} \in \mathbb{Z}^{d}: \mathbf{z} \neq 0}|U(\mathbf{z})|\|\mathbf{z}\| .
$$

Очевидны следующие неравенства:

$$
\begin{aligned}
\left|\mathrm{H}_{\Lambda}[\rho, \mathbf{s}]-\hat{\mathbf{H}}_{\Lambda}[\rho, \mathbf{s}]\right| \leq \frac{1}{2} \sum_{\substack{\mathbf{x} \in \Lambda, \mathbf{y} \in \mathbb{Z}^{d} \backslash \Lambda}}|U(\mathbf{x}-\mathbf{y})|\left|I\left(\mathbf{s}(\mathbf{x}), \mathbf{s}\left(\mathrm{P}_{\Lambda} \mathbf{y}\right)\right)\right| \leq \\
\quad \leq \frac{I}{2} \sum_{\substack{\mathbf{x} \in \Lambda, \mathbf{y} \in \mathbb{Z}^{d} \backslash \Lambda}}|U(\mathbf{x}-\mathbf{y})|=\frac{I}{2} \sum_{z \in \mathbb{Z}^{d}: \mathbf{z} \neq 0}|U(\mathbf{z})||\Gamma(\mathbf{z} ; \Lambda)|,
\end{aligned}
$$

где $\Gamma(\mathbf{z} ; \Lambda)=\{\langle\mathbf{x}, \mathbf{y}\rangle: \mathbf{x}-\mathbf{y}=\mathbf{z}, \mathbf{x} \in \Lambda, \mathbf{y} \notin \Lambda\}$.

Каждая пара, принадлежащая $\Gamma(\mathbf{z}, \Lambda)$, взаимно однозначным образом определяется точкой $\mathbf{x} \in \Lambda$ так, что $\mathbf{y}=\mathbf{x}-\mathbf{z} \notin \Lambda$. При этом $\mathbf{x}=\mathbf{y}+\mathbf{z} \in \mathbb{Z}^{d} \backslash \Lambda+\mathbf{z}$. Следовательно,

$$
\{\mathbf{x}:\langle\mathbf{x}, \mathbf{y}\rangle \in \Gamma(\mathbf{z}, \Lambda)\} \subset\left\{\mathbf{x}: \mathbf{x} \in \Lambda, \mathbf{x} \in \mathbb{Z}^{d} \backslash \Lambda+\mathbf{z}\right\}=\Lambda \cap\left(\mathbb{Z}^{d} \backslash \Lambda+\mathbf{z}\right) .
$$

Тогда $|\Gamma(\mathbf{z}, \Lambda)| \leq\left|\Lambda \cap\left(\left(\mathbb{Z}^{d} \backslash \Lambda\right)+\mathbf{z}\right)\right|$. Поэтому, применив оценку (14), получаем (15).

Из полученной оценки (15) разности энергий следует, что для дальнодействующих взаимодействий с конечной нормой $\|\cdot\|_{0}$, для которых

$$
\sum_{\mathbf{x} \in \mathbb{Z}^{d}}|U(\mathbf{x})|\|\mathbf{x}\|=\infty
$$

разность (15) возрастает быстрее, чем площадь поверхности $\Lambda$. Это затрудняет использование аппроксимации исходной системы соответствующей ей системой с периодическими граничными условиями в случае дальнодействующих потенциалов взаимодействия. Этот факт был отмечен в работах [Клюев, Вирченко 2015], [Вирченко, 1991]. Тем не менее, справедливо 
Следствие. $Е с л и \mathrm{H}_{\Lambda}[\rho, \mathrm{s}]$ имеет конечную норму, то при термодинамическом предельном переходе имеет место

$$
\lim _{|\Lambda| \rightarrow \infty} \frac{1}{|\Lambda|}\left|\mathrm{H}_{\Lambda}[\rho, \mathbf{s}]-\hat{\mathrm{H}}_{\Lambda}[\rho, \mathbf{s}]\right|=0 .
$$

$\square$ Так как $\left\|\mathbf{H}_{\Lambda}[\rho, \mathbf{s}]\right\|_{0}<\infty$, то $\sum_{\mathbf{z} \in \mathbb{Z}^{d}}|U(\mathbf{z})|<\infty$. Для функций $U(\cdot)$ такого типа имеет место

$$
\lim _{L \rightarrow \infty} \frac{1}{L} \sum_{\mathbf{z} \in \Lambda}|U(\mathbf{z})|\|\mathbf{z}\|=0 .
$$

В самом деле, выберем произвольное число $\varepsilon>0$ и найдем такой размер $L_{\varepsilon}$, для которого

$$
\sum_{\mathbf{z}:|\mathbf{z}|>L_{\varepsilon}}|U(\mathbf{z})|<\varepsilon
$$

Тогда при $L>L_{\varepsilon}$ справедлива оценка

$$
\sum_{\mathbf{z} \in \Lambda}|U(\mathbf{z})|\|\mathbf{z}\|=\sum_{\mathbf{z} \in \Lambda:|\mathbf{z}|>L_{\varepsilon}}|U(\mathbf{z})|\|\mathbf{z}\|+\sum_{\mathbf{z} \in \Lambda:|\mathbf{z}| \leq L_{\varepsilon}}|U(\mathbf{z})|\|\mathbf{z}\| .
$$

Для оценки первой суммы используем неравенство $\|\mathbf{z}\|<L$ при фиксированном $L_{\varepsilon}$,

$$
\sum_{\mathbf{z} \in \Lambda}|U(\mathbf{z})|\|\mathbf{z}\|<\varepsilon L+\sum_{\mathbf{z} \in \Lambda:|\mathbf{z}| \leq L_{\varepsilon}}|U(\mathbf{z})|\|\mathbf{z}\|
$$

Подставим эту оценку в (15), где учтем, что при $|\mathbf{z}|>L \sqrt{d}+1, \Lambda \cap\left(\left(\mathbb{Z}^{d} \backslash \Lambda\right)+\mathbf{z}\right)=\varnothing$,

$$
\begin{aligned}
\left|\mathrm{H}_{\Lambda}[\rho, \mathbf{s}]-\hat{\mathbf{H}}_{\Lambda}[\rho, \mathbf{s}]\right|< & \frac{1}{2} d(L+1)^{d-1} I \sum_{\substack{\mathbf{x} \in \mathbb{Z}^{d}: \mathbf{z} \neq 0 \\
|\mathbf{z}| \leq L \sqrt{d}+1}}|U(\mathbf{z})|\|\mathbf{z}\|< \\
& <\frac{1}{2} d L^{d} I \varepsilon+\frac{1}{2} d(L+1)^{d-1} I \sum_{\substack{\mathbf{x} \in \mathbb{Z}^{d}: \mathbf{z} \neq 0,|\mathbf{z}| \leq L_{\varepsilon} \\
|\mathbf{z}| \leq L \sqrt{d}+1}}|U(\mathbf{z})|\|\mathbf{z}\|,
\end{aligned}
$$

так как $L \sqrt{d}+1>L_{\varepsilon}$. Поделим обе части неравенства на $|\Lambda|=L^{d}$ и перейдем к пределу $L \rightarrow \infty$. В результате, так как второе слагаемое в правой части стремится к нулю при фиксированной величине $L_{\varepsilon}$, то мы получим, что

$$
\lim _{|\Lambda| \rightarrow \infty} \frac{1}{|\Lambda|}\left|\mathrm{H}_{\Lambda}[\rho, \mathbf{s}]-\hat{\mathrm{H}}_{\Lambda}[\rho, \mathbf{s}]\right| \leq \varepsilon .
$$

Ввиду произвольности величины $\varepsilon>0$, получаем (17).

4. Интегральные уравнения для плотностей $f_{m}$. Выведем систему уравнений для плотностей $f_{m}^{(\Lambda)}(X ; \mathbf{s}(\mathbf{x}), \mathbf{x} \in X)$ модели решеточного газа с парным потенциалом $U$, аналогичную системе, введенной в работе [Пастур, 1974], при изучении модели решеточного газа. При ее выводе используется схема рассуждений, аналогичная той (см. [Рюэль, 1971]), которая используется для анализа многочастичных конфигурационных функций в статистической механике непрерывных систем.

Пусть $X=\{\mathbf{x}: \rho(\mathbf{x})=1\} \subset \Lambda$. Воспользовавшись формулой (12), запишем выражение (7) для плотностей $f_{m}^{(\Lambda)}(X ; \mathbf{s}(\mathbf{x}), \mathbf{x} \in X)$ в следующем виде:

$$
\begin{aligned}
f_{m}^{(\Lambda)}(X ; \mathbf{s}(\mathbf{x}), \mathbf{x} \in X) & =Q_{\Lambda}^{-1} \sum_{Y \subset \Lambda \backslash X}\left(\prod_{\mathbf{x} \in X} w(s(\mathbf{x}))\right) \times \\
& \times \int_{\substack{\mathbf{s}(\mathbf{y}) \in \mathbb{R}^{n} \\
\mathbf{y} \in Y}} \exp \left(-\mathrm{H}_{\Lambda}(X \cup Y ; \mathbf{s}) / T\right) \prod_{\mathbf{y} \in Y} w(s(\mathbf{y})) \mathrm{d} \mathbf{s}(\mathbf{y}) .
\end{aligned}
$$

Введем в рассмотрение функцию $K\left(\mathbf{x}_{1}-\mathbf{x}_{2} ; \mathbf{s}_{1}, \mathbf{s}_{2}\right)=\exp \left(-U\left(\mathbf{x}_{1}-\mathbf{x}_{2}\right) I\left(\mathbf{s}_{1}, \mathbf{s}_{2}\right) / T\right)-1$, определенную для каждых $\mathbf{x}_{1}, \mathbf{x}_{2} \in \mathbb{Z}^{d}, \mathbf{s}_{1}, \mathbf{s}_{2} \in \mathbb{R}^{n}$, а также следующую функцию на $\mathbb{Z}^{d} \times \mathfrak{P}\left(\mathbb{Z}^{d}\right)$, где $\mathfrak{P}\left(\mathbb{Z}^{d}\right)$ семейство всех конечных подмножеств $\mathbb{Z}^{d}$ :

$$
W_{\Lambda}(\mathbf{x} ; X ; \mathbf{s}(\mathbf{y}), \mathbf{y} \in X)=\exp \left(\left[(\mathbf{s}(\mathbf{x}), \mathbf{h})-\sum_{\substack{\mathbf{x} \in X, \mathbf{y} \in \mathbb{Z}^{d}: \\ \mathbf{x} \neq \mathbf{P}_{\Lambda} \mathbf{y} \in X}} U(\mathbf{x}-\mathbf{y}) I\left(\mathbf{s}(\mathbf{x}), \mathbf{s}\left(\mathrm{P}_{\Lambda} \mathbf{y}\right)\right)\right] / T\right),
$$


$W_{\Lambda}(\mathbf{x} ; \varnothing)=1$. Тогда для любых $X \subset \Lambda \backslash\{\mathbf{x}\}, Y \subset \Lambda \backslash\{\mathbf{x}\}, X \cap Y=\varnothing$ имеет место

$$
\begin{aligned}
\exp \left(-\mathrm{H}_{\Lambda}(\{\mathbf{x}\} \cup X \cup Y ; \mathbf{s}) / T\right)= \\
\quad=W_{\Lambda}(\mathbf{x} ; X ; \mathbf{s}(\mathbf{y}), \mathbf{y} \in X) W_{\Lambda}(\mathbf{x} ; Y ; \mathbf{s}(\mathbf{y}), \mathbf{y} \in Y) \exp \left(-\mathrm{H}_{\Lambda}(X \cup Y ; \mathbf{s}) / T\right)= \\
=W_{\Lambda}(\mathbf{x} ; X ; \mathbf{s}(\mathbf{y}), \mathbf{y} \in X) \prod_{\mathbf{y} \in Y}(1+K(\mathbf{x}-\mathbf{y} ; \mathbf{s}(\mathbf{x}), \mathbf{s}(\mathbf{y}))) \exp \left(-\mathrm{H}_{\Lambda}(X \cup Y ; \mathbf{s}) / T\right) .
\end{aligned}
$$

Это равенство сохраняется и при $|X \cup Y|=1$, так как в этом случае $\mathrm{H}_{\Lambda}(X \cup Y ; \mathbf{s})=\exp ((\mathbf{h}, \mathbf{s}(\mathbf{z})))$, где $\mathbf{z}=\mathbf{x}, \mathbf{y}$, в зависимости от того, какое из множеств пусто.

Определим, далее, функцию

$$
K(\mathbf{x}, \mathbf{s} ; Y ; \mathbf{s}(y), \mathbf{y} \in Y)=\left\{\prod_{\mathbf{y} \in Y} K(\mathbf{x}-\mathbf{y} ; \mathbf{s}, \mathbf{s}(\mathbf{y}), \mathbf{y} \in Y), \text { при }|Y|>0 ; 1, \text { при }|Y|=0 .\right\}
$$

такую, что

$$
\prod_{\mathbf{y} \in Z}(1+K(\mathbf{x}-\mathbf{y} ; \mathbf{s}(\mathbf{x}), \mathbf{s}(\mathbf{y})))=\sum_{Y \subset Z} \prod_{\mathbf{y} \in Y} K(\mathbf{x}-\mathbf{y} ; \mathbf{s}(\mathbf{x}), \mathbf{s}(\mathbf{y}))=\sum_{Y \subset Z} K(\mathbf{x}, \mathbf{s} ; Y ; \mathbf{s}(y), \mathbf{y} \in Y) .
$$

Наконец, введем в рассмотрение пространство $\mathfrak{E}_{\Lambda}$ всех наборов $\mathrm{f}^{\lambda}=\left\langle f_{m}^{(\Lambda)}(X ; \mathbf{s}(\mathbf{x}), \mathbf{x} \in X): \varnothing \neq\right.$ $X \subset \Lambda\rangle$ функций с $\mathbf{s}(\mathbf{x}) \in \mathbb{R}^{n}, \mathbf{x} \in \Lambda$.

Подставим (19), вместе с (20), в выражение для $f_{m+1}^{(\Lambda)}(X \cup\{\mathbf{x}\} ; \mathbf{s}(\mathbf{y}), \mathbf{y} \in X \cup\{x\})$ с $X \subset \Lambda \backslash\{\mathbf{x}\}$, получаемое на основе (18):

$$
\begin{aligned}
& f_{m+1}^{(\Lambda)}(X \cup\{\mathbf{x}\} ; \mathbf{s}(\mathbf{y}), \mathbf{y} \in X \cup\{\mathbf{x}\})=w(\mathbf{s}(\mathbf{x})) W_{\Lambda}(\mathbf{x} ; X ; \mathbf{s}(\mathbf{y}), \mathbf{y} \in X) \times \\
& \times Q_{\Lambda}^{-1} \sum_{Y \subset \Lambda \backslash X \cup\{\mathbf{x}\}} \sum_{\substack{Z: Y \subset Z, Z \subset \Lambda \backslash X \cup\{\mathbf{x}\}}}\left(\prod_{\mathbf{y} \in X} w(s(\mathbf{y}))\right) \int_{\substack{\mathbf{s}(\mathbf{y}) \in \mathbb{R}^{n}: \\
\mathbf{y} \in Y}} K(\mathbf{x}, \mathbf{s} ; Y ; \mathbf{s}(y), \mathbf{y} \in Y) \times \\
& \\
& \quad \times \exp \left(-\mathrm{H}_{\Lambda}(X \cup Y ; \mathbf{s}) / T\right) \prod_{\mathbf{y} \in Y} w(s(\mathbf{y})) \mathrm{d} \mathbf{s}(\mathbf{y})
\end{aligned}
$$

и произведем следующие преобразования суммы:

$$
\begin{aligned}
& Q_{\Lambda}^{-1} \sum_{Y \subset \Lambda \backslash X \cup\{\mathbf{x}\}} \sum_{\substack{Z: Y \subset Z, Z \subset \Lambda \backslash X \cup\{\mathbf{x}\}}}\left(\prod_{\mathbf{y} \in X} w(s(\mathbf{y}))\right) \int_{\substack{\mathbf{s}(\mathbf{y}) \in \mathbb{R}^{n}: \\
\mathbf{y} \in Y}} K(\mathbf{x}, \mathbf{s} ; Y ; \mathbf{s}(y), \mathbf{y} \in Y) \times \\
& \times \exp \left(-\mathrm{H}_{\Lambda}(X \cup Y ; \mathbf{s}) / T\right) \prod_{\mathbf{y} \in Y} w(s(\mathbf{y})) \mathrm{d} \mathbf{s}(\mathbf{y})= \\
& =\sum_{Y \subset \Lambda \backslash X \cup\{\mathbf{x}\}} \int_{\substack{\mathbf{s}(\mathbf{y}) \in \mathbb{R}^{n}: \\
\mathbf{y} \in Y}} K(\mathbf{x}, \mathbf{s} ; Y ; \mathbf{s}(y), \mathbf{y} \in Y) \prod_{\mathbf{y} \in X \cup Y} w(s(\mathbf{y})) \mathrm{d} \mathbf{s}(\mathbf{y}) \times \\
& \times Q_{\Lambda}^{-1} \sum_{Z \subset \Lambda \backslash(X \cup Y \cup\{\mathbf{x}\})} \int_{\substack{\mathbf{s}(\mathbf{z}) \in \mathbb{R}^{n}: \\
\mathbf{z} \in \Lambda Z}} \exp \left(-\mathrm{H}_{\Lambda}(X \cup Y \cup Z ; \mathbf{s}) / T\right) \prod_{\mathbf{z} \in Z} w(s(\mathbf{z})) \mathrm{d} \mathbf{s}(\mathbf{z})= \\
& =\sum_{Y \subset \Lambda \backslash(X \cup\{\mathbf{x}\})} \int_{\substack{\mathbf{s}(\mathbf{y}) \in \mathbb{R}^{n}: \\
\mathbf{y} \in Y}} K(\mathbf{x}, \mathbf{s} ; Y ; \mathbf{s}(y), \mathbf{y} \in Y) \times \\
& \times\left[f_{m+|Y|}^{(\Lambda)}(X \cup Y ; \mathbf{s}(\mathbf{z}), \mathbf{z} \in X \cup Y)-f_{m+1+|Y|}^{(\Lambda)}(\{\mathbf{x}\} \cup X \cup Y ; \mathbf{s}(\mathbf{z}), \mathbf{z} \in X \cup Y \cup\{\mathbf{x}\})\right] \prod_{\mathbf{y} \in Y} \mathrm{~d} \mathbf{s}(\mathbf{y}),
\end{aligned}
$$

так как $\sum_{Z \subset \Lambda \backslash(\{\mathbf{x}\} \cup X \cup Y)}(\cdot)=\sum_{Z \subset \Lambda \backslash(X \cup Y)}(\cdot)-\sum_{\mathbf{x} \in Z \subset \Lambda \backslash(X \cup Y)}(\cdot)$. Подставим полученное выражение в (21). Выделив слагаемые с $Y=\varnothing$, получаем систему линейных алгебраических тождеств набора плотностей распределения вероятностей $f_{m}^{(\Lambda)}(X ; \mathbf{s}(\mathbf{x}), \mathbf{x} \in X)$ с $\mathbf{s}(\mathbf{x}) \in \mathbb{R}^{n}, \mathbf{x} \in X, \varnothing \neq X \subset \Lambda$ :

$$
f_{m+1}^{(\Lambda)}(X \cup\{\mathbf{x}\} ; \mathbf{s}(\mathbf{y}), \mathbf{y} \in X \cup\{\mathbf{x}\})=w(\mathbf{s}(\mathbf{x})) W_{\Lambda}(\mathbf{x} ; X ; \mathbf{s}(\mathbf{y}), \mathbf{y} \in X) \times
$$




$$
\begin{gathered}
\times\left[f_{m}^{(\Lambda)}(X ; \mathbf{s}(\mathbf{z}), \mathbf{z} \in X)-f_{m+1}^{(\Lambda)}(\{\mathbf{x}\} \cup X ; \mathbf{s}(\mathbf{z}), \mathbf{z} \in X \cup\{\mathbf{x}\})+\right. \\
+\sum_{Y \subset \Lambda \backslash(X \cup\{\mathbf{x}\})} \int_{\substack{\mathbf{s}(\mathbf{y}) \in \mathbb{R}^{n} \\
\mathbf{y} \in Y}} K(\mathbf{x}, \mathbf{s} ; Y ; \mathbf{s}(y), \mathbf{y} \in Y) \times \\
\left.\times\left[f_{m+|Y|}^{(\Lambda)}(X \cup Y ; \mathbf{s}(\mathbf{z}), \mathbf{z} \in X \cup Y)-f_{m+1+|Y|}^{(\Lambda)}(\{\mathbf{x}\} \cup X \cup Y ; \mathbf{s}(\mathbf{z}), \mathbf{z} \in X \cup Y \cup\{\mathbf{x}\})\right] \prod_{\mathbf{y} \in Y} \mathrm{~d} \mathbf{s}(\mathbf{y})\right] .
\end{gathered}
$$

Таким образом, нами получена система интегральных уравнений Фредгольма 2-го рода, которой подчинен набор плотностей распределения для векторных решетчатых моделей.

Можно показать, что эту систему можно рассматривать в пространстве $\mathfrak{E}_{\Lambda}$. В связи с этим, ее вид не зависит от выбора точки $\mathbf{x}$ в каждом из множеств $X \cup\{\mathbf{x}\} \subset \Lambda$. Поэтому ее выбор можно унифицировать. Будем полагать, что в каждом $X \subset \Lambda$ эта точка выбирается первой в смысле лексикографического порядка на решетке $\mathbb{Z}^{d}$.

Наконец, укажем, что предельные плотности $f_{m}(X ; \mathbf{s}(\mathbf{y}), \mathbf{y} \in X), m \in \mathbb{N}, \varnothing \neq X \subset \mathbb{Z}^{d}$, получаемые при термодинамическом предельном переходе $\Lambda \rightarrow \mathbb{Z}^{d}$, должны удовлетворять следующей предельной системе интегральных уравнений:

$$
\begin{aligned}
& \quad f_{m+1}(X \cup\{\mathbf{x}\} ; \mathbf{s}(\mathbf{y}), \mathbf{y} \in X \cup\{\mathbf{x}\})=w(\mathbf{s}(\mathbf{x})) W(\mathbf{x} ; X ; \mathbf{s}(\mathbf{y}), \mathbf{y} \in X) \times \\
& \quad \times\left[f_{m}(X ; \mathbf{s}(\mathbf{z}), \mathbf{z} \in X)-f_{m+1}(\{\mathbf{x}\} \cup X ; \mathbf{s}(\mathbf{z}), \mathbf{z} \in X \cup\{\mathbf{x}\})+\right. \\
& +\sum_{Y \subset \mathbb{Z}^{d} \backslash(X \cup\{\mathbf{x}\})} \int_{\substack{\mathbf{s}(\mathbf{y}) \in \mathbb{R}^{n} \\
\mathbf{y} \in Y}} K(\mathbf{x}, \mathbf{s} ; Y ; \mathbf{s}(y), \mathbf{y} \in Y) \times \\
& \left.\times\left[f_{m+|Y|}(X \cup Y ; \mathbf{s}(\mathbf{z}), \mathbf{z} \in X \cup Y)-f_{m+1+|Y|}(\{\mathbf{x}\} \cup X \cup Y ; \mathbf{s}(\mathbf{z}), \mathbf{z} \in X \cup Y \cup\{\mathbf{x}\})\right] \prod_{\mathbf{y} \in Y} \mathrm{~d} \mathbf{s}(\mathbf{y})\right] .
\end{aligned}
$$

Эта система может рассматриваться как видоизменение, по отношению к классическим векторным решетчатым моделям, известной системы интегральных уравнений Кирквуда - Зальцбурга [Kirkwood, Salsburg, 1953] в статистической механике непрерывных моделей.

\section{Список литературы}

1. Ахиезер А. И., Барьяхтар В. Г., Пелетминский С. В. 1967. Спиновые волны. М., Наука, 368.

2. Вирченко Ю. П. 1991. К теории основного состояния обменной модели Гейзенберга. Проблемы теоретической физики. Киев: Наукова думка, 80-96.

3. Добрушин Р. Л. 1968. Гиббсовские случайные поля для решетчатых систем с попарным взаимодействием. Функциональный анализ и его приложения, 4(1): 31-43.

4. Клюев А. С., Вирченко Ю. П. 2015. Оценка энергии векторной решеточной модели с периодическими граничными условиями. Научные ведомости Белгородского государственного университета. Серия: Математика. Физика, 11(208)(39): 121-125.

5. Минлос Р. А. 1968. Лекции по статистической физике. Успехи мат. наук, 1: 133-190.

6. Минлос Р. А. 2002. Введение в математическую статистическую физику. М., МЦНМО, 111.

7. Пастур Л. А. 1974. Спектральная теория уравнений Кирквуда - Зальцбурга в конечном объеме. Теорет. и матем. физика, 18(2): 233-242.

8. Рюэль Д. 1971. Статистическая механика. Строгие результаты. М., Мир, 367.

9. Gallavotti G., Miracle-Sole S. 1967. Statistical Mechanics of Lattice Systems. Commun. Math. Phys, 5: $317-323$.

10. Gallavotti G. Statistical mechanics. 1999. Roma: Dipartimento di Fisica Universitá di Roma, 349.

11. de Lacheisserie E., Gignoux D., Schlenker M. 2005. Magnetism: Fundamentals. Springer, 1: 315-317.

12. Kirkwood J. G., Salsburg Z. 1953. W. The statistical mechanical theory of molecular distribution functions in liquids. Discussion of the Faraday Society, 15(1): 28-34. 
13. Stohr J., Siegmann H. C. 2006. Magnetism: From Fundamentals to Nanoscale Dynamics. Berlin, Heidelberg: Springer-Verlag, 290-293.

\section{References}

1. Akhiezer A. I., Baryakhtar V. G., Peletminsky S. V. 1967. Spinovye volny [Spin waves]. M., Nauka, 368 .

2. Virchenko Yu. P. 1991. K teorii osnovnogo sostoyaniya obmennoj modeli Gejzenberga [On the theory of the ground state of the Heisenberg exchange model]. Problemy teoreticheskoj fiziki. Kiev: Naukova dumka, 80-96.

3. Dobrushin R. L. 1968. Gibbsovskie sluchajnye polya dlya reshetchatyh sistem s poparnym vzaimodejstviem [Gibbs random fields for lattice systems with pairwise interaction]. Funkcional'nyj analiz i ego prilozheniya, 4(1): 31-43.

4. Klyuev A. S., Virchenko Yu. P. 2015. Ocenka energii vektornoj reshetochnoj modeli s periodicheskimi granichnymi usloviyami [Energy estimation of a vector lattice model with periodic boundary conditions]. Nauchnye vedomosti Belgorodskogo gosudarstvennogo universiteta. Seriya: Matematika. Fizika, 11(208)(39): 121-125.

5. Minlos R. A. 1968. Lekcii po statisticheskoj fizike [Lectures on statistical physics]. Uspekhi mat. nauk, 1: 33-190.

6. Minlos R. A. 2002. Vvedenie v matematicheskuyu statisticheskuyu fiziku [Introduction to mathematical statistical physics]. M., MCNMO, 111.

7. Pastur L. A. 1974. Spektral'naya teoriya uravnenij Kirkvuda-Zal'cburga v konechnom ob"eme [The spectral theory of the Kirkwood - Salzburg equations within the corresponding limits]. Teoret. i matem. fizika, 18(2): 233-242.

8. Ruelle D. 1969. Statistical Mechanics. Rigorous Results. New York-Amsterdam: W. A. Benjamin, Inc, 367 .

9. Gallavotti G., Miracle-Sole S. 1967. Statistical Mechanics of Lattice Systems. Commun. Math. Phys. 5: 317-323.

10. Gallavotti G. Statistical mechanics. 1999. Roma: Dipartimento di Fisica Universitá di Roma, 349.

11. de Lacheisserie E., Gignoux D., Schlenker M. 2005. Magnetism: Fundamentals. Springer, 1: 315-317.

12. Kirkwood J. G., Salsburg Z. 1953. W. The statistical mechanical theory of molecular distribution functions in liquids. Discussion of the Faraday Society. 15(1): 28-34.

13. Stohr J., Siegmann H. C. 2006. Magnetism: From Fundamentals to Nanoscale Dynamics. Berlin, Heidelberg: Springer-Verlag, 290-293.

Получена 01.02.2020

Вирченко Юрий Петрович - доктор физико-математических наук, доцент, профессор кафедры теоретической и математической физики института инженерных и цифровых технологий Белгородского государственного национального исследователького университета

ул. Победы, 85, г. Белгород, Россия, 308015

E-mail: virch@bsu.edu.ru

Московченко Екатерина Юрьевна - аспирантка первого года обучения кафедры прикладной математики и компьютерного моделирования института инженерных и цифровых технологий Белгородского государственного национального исследователького университета

ул. Победы, 85, г. Белгород, Россия, 308015

E-mail: 1079708@bsu.edu.ru 